\title{
Publisher Correction: The determinants of consciousness of human faces
}

Yaniv Abir (D), Asael Y. Sklar, Ron Dotsch, Alexander Todorov and Ran R. Hassin (D)

Correction to: Nature Human Behaviour https://doi.org/10.1038/s41562-017-0266-3, published online 18 December 2017.

In the version of this Letter originally published, the image of the face in Fig. 1c was mistakenly visible but should have been blended into the pattern. This has now been corrected. 